\title{
Dynamics, nucleosynthesis, and kilonova signature of black hole - neutron star merger ejecta
}

\author{
Rodrigo Fernández ${ }^{1,2,3}$, Francois Foucart ${ }^{4,5}$, \\ Daniel Kasen ${ }^{2,3,4}$, Jonas Lippuner ${ }^{6}$, Dhruv Desai ${ }^{2}$, \\ Luke F. Roberts ${ }^{7}$ \\ ${ }^{1}$ Department of Physics, University of Alberta, Edmonton, AB T6G 2E1, \\ Canada. \\ 2 Department of Physics, University of California, Berkeley, CA 94720, USA. \\ 3 Department of Astronomy \& Theoretical Astrophysics Center, University of \\ California, Berkeley, CA 94720, USA. \\ ${ }^{4}$ Nuclear Science Division, Lawrence Berkeley National Laboratory, Berkeley, \\ CA 94720, USA. \\ ${ }^{5}$ NASA Einstein Fellow \\ 6 TAPIR, Walter Burke Institute for Theoretical Physics, California Institute of \\ Technology, Pasadena, CA 99125, USA. \\ 7 National Superconducting Cyclotron Laboratory and Department of Physics \\ and Astronomy, Michigan State University, East Lansing, MI 48824, USA \\ E-mail: rafernan@ualberta.ca
}

\begin{abstract}
We investigate the ejecta from black hole - neutron star mergers by modeling the formation and interaction of mass ejected in a tidal tail and a disk wind. The outflows are neutron-rich, giving rise to optical/infrared emission powered by the radioactive decay of $r$-process elements (a kilonova). Here we perform an end-to-end study of this phenomenon, where we start from the output of a fully-relativistic merger simulation, calculate the post-merger hydrodynamical evolution of the ejecta and disk winds including neutrino physics, determine the final nucleosynthetic yields using post-processing nuclear reaction network calculations, and compute the kilonova emission with a radiative transfer code. We study the effects of the tail-to-disk mass ratio by scaling the tail density. A larger initial tail mass results in fallback matter becoming mixed into the disk and ejected in the subsequent disk wind. Relative to the case of a disk without dynamical ejecta, the combined outflow has lower mean electron fraction, faster speed, larger total mass, and larger absolute mass free of high-opacity Lanthanides or Actinides. In most cases, the nucleosynthetic yield is dominated by the heavy $r$-process contribution from the unbound part of the dynamical ejecta. A Solarlike abundance distribution can however be obtained when the total mass of the dynamical ejecta is comparable to the mass of the disk outflows. The kilonova has a characteristic duration of 1 week and a luminosity of $\sim 10^{41} \mathrm{erg} \mathrm{s}^{-1}$, with orientation effects leading to variations of a factor $\sim 2$ in brightness. At early times $(<1$ day) the emission includes an optical component from the (hot) Lanthanide-rich material, but the spectrum evolves quickly to the infrared thereafter.
\end{abstract}

Keywords: accretion, accretion disks - dense matter - gravitational waves hydrodynamics - neutrinos - nuclear reactions, nucleosynthesis, abundances. 


\section{Introduction}

The recent success of Advanced LIGO in detecting gravitational waves (GWs) from binary black hole (BH-BH) mergers [1, 2] has marked the onset of GW Astronomy. As the sensitivity of detectors improves, detection of double neutron star (NS-NS) and NS-BH mergers in GWs is expected within the next few years [3]. Mergers that involve NSs are of interest to a broad community because they can help to test the equation of state (EOS) of dense matter (e.g., [4), are a prime candidate astrophysical site for $r$-process elements (e.g., [5, 6, 7), and are expected to produce electromagnetic (EM) counterparts over a wide range of timescales and wavelengths (e.g., [8, 9]).

The most easily detectable EM counterpart of NS-NS/NS-BH mergers is a supernova-like transient powered by the radioactive decay of $r$-process elements produced in the expanding ejecta, commonly known as a kilonova or macronova [10, 11, 12, 13, 14. These transients arise from the sub-relativistic ejecta of the merger and are hence not affected by beaming like short gamma-ray bursts (SGRBs). The emission is detectable at optical and infrared wavelengths [15].

The intimate relation between a kilonova and the production of $r$-process elements makes the transient a powerful diagnostic of the physical conditions in the merger. This diagnostic power arises from the sensitivity of the optical opacity to the type of $r$-process composition of the ejecta: even a small fraction of Lanthanides or Actinides (mass number $A \gtrsim 140$ ) can increase the optical opacity by orders of magnitude relative to iron-group-like composition [16, 17. The resulting kilonova can range from an optical transient lasting days, to an infrared transient lasting for weeks [18, 19, 20, 21, 22.

Two distinct sources of merger ejecta with potentially different compositions contribute to the kilonova emission. Material expelled on a dynamical time leaves the system first, with typical velocities $0.1-0.3 c$ (e.g., 23]). For NS-BH mergers, ejection is driven mainly by tidal forces, and therefore the ejecta is launched primarily on the equatorial plane. For NS-NS mergers additional ejection occurs from the contact interface, leading to an outflow more widely distributed in solid angle. The tidally ejected material is mostly neutron-rich, generating a robust abundance of elements with $A>130$ that follow the Solar System $r$-process distribution [24, 25]. A shockheated and/or neutrino-irradiated component of the interface ejecta can have high electron fraction $Y_{e}$, by virtue of weak interactions, thereby producing lighter $r$ process elements 26. The prevalence of the high- $Y_{e}$ component is an open research question, however, as it is sensitive to the treatment of neutrinos and the nuclear EOS [27, 28, 29, 6, 30, 31, 32.

The second source of ejecta is the remnant accretion disk. Outflows can be launched on the thermal time $(\sim 10-100 \mathrm{~ms})$ if there is sufficient neutrino irradiation from a hypermassive NS [33, 34, 35], or on longer times $\gtrsim 1$ s once neutrino emission has subsided and the disk reaches the advective state [36, 37, 38, 5]. Magneticallydriven disk winds can also contribute to these disk outflows at early times during the transient phase (e.g. [39]) or in the late disk evolution. The disk ejecta component is slower (velocity $\sim 0.05 \mathrm{c}$ ) than the dynamical ejecta, and is generally less neutronrich due to longer exposure to weak interactions. The resulting composition can be dominated by light $r$-process elements. Whether heavier elements $(A>130)$ are also produced depends on astrophysical parameters such as the disk mass or the properties of angular momentum transport [5, 7. The amount of mass ejected can be comparable or even dominate the dynamical ejecta, depending on the binary parameters (e.g., 9]). 
The different compositions and kinematic properties of these two ejecta channels have implications for the magnitude, color, and duration of the kilonova: opacity and velocity control the diffusion time. Furthermore, the expected geometry of NS-BH merger ejecta is such that the kilonova color can have a strong viewing angle dependency [40. Likewise, the dynamical ejecta has a gravitationally bound component that falls back onto the central object, potentially interacting with the disk outflow and hence altering the net composition and mass ejection from the disk. A first attempt at characterizing the interplay between these two ejecta components was made by [41] for the case of NS-BH mergers. Starting from the output of a Newtonian merger simulation, the long-term evolution of all components was followed until homology was reached. The disk outflow was found to suppress fallback accretion relative to the case in which the disk is absent, with long-term engine activity still possible by accretion from the disk. No significant changes in the disk outflow properties were found relative to the case without dynamical ejecta, although only one realization was studied in which the disk has a high mass $\left(0.2 M_{\odot}\right)$ relative to the bound dynamical ejecta $\left(0.02 M_{\odot}\right)$.

Here we improve upon the work of 41 by using initial conditions mapped from a fully relativistic NS-BH simulation that includes neutrino irradiation and therefore provides more realistic initial conditions. In addition, we explore the effect of varying the relative initial masses of dynamical ejecta and disk, by scaling the initial dynamical ejecta density. Given that the dynamical ejecta moves nearly ballistically, this is a good first approximation to test the impact the ratio of dynamical- to disk ejecta without having to perform a large number of costly merger simulations. We conduct the post-merger simulations in axisymmetry, and include the dominant energy and lepton number source terms, in addition to passive tracer particles. The output is postprocessed with a nuclear reaction network and a radiative transfer code to compute $r$-process nucleosynthesis yields and kilonova light curve and spectral predictions, respectively.

The structure of this paper is the following. Section 2 describes our computational method, Section 3 presents the results of our hydrodynamic simulations of the remnant evolution, Section 4 describes our nucleosynthesis results, Section 5 our kilonova predictions, and Section 6 presents our summary and discussion.

\section{Methods}

\subsection{Initial conditions}

The initial condition for our models is the final snapshot of a BH-NS post-merger remnant simulation presented in [28]. This simulation evolves the accretion disk resulting from the merger of a $7 M_{\odot} \mathrm{BH}$ with a $1.4 M_{\odot} \mathrm{NS}$ (case M14-7-S8 from [42]), up to $20 \mathrm{~ms}$ after the merger. The dimensionless spin of the $\mathrm{BH}$ before merger is $\chi=0.8$, aligned with the orbital angular momentum of the system. After merger, the $\mathrm{BH}$ spin is $\chi=0.861$. The inspiral, merger, and post-merger evolution are performed using the SpEC code [43, which evolves Einstein's equations of general relativity in the Generalized Harmonic formalism using pseudospectral methods [44, the relativistic equations of hydrodynamics in conservative form using high-order shock capturing finite volume methods [45, 46, and the first two moments of the neutrino distribution function with an analytical M1 closure [47, 48, 28]. The equation of state for the neutron star is that of Lattimer and Swesty (1991) [49, using a compressibility 
parameter $K_{0}=220 \mathrm{MeV}$. The computational domain in SpEC encompasses the central object and the accretion disk, while dynamical ejecta with a fallback time longer than $\sim 20 \mathrm{~ms}$ is allowed to leave the domain. Up to the disruption of the neutron star by the gravitational field of the black hole, the SpEC evolution explicitly imposes symmetry of the fluid variables across the equatorial plane, while the disk formation and post-merger evolution is performed without that symmetry requirement (although SpEC preserves quite accurately the original symmetry of the system). We note that while for the system studied here the exact solution is symmetric across the equatorial plane, explicitly imposing this condition in simulations can create some numerical artifacts in the results. In particular, small errors in the vertical component of the fluid velocity in the equatorial plane can push material away from that plane, leading to density maxima offset from the equator. While this effect decreases as the numerical resolution increases, it can be easily observed in lower-resolution regions e.g. the low density regions of the tidal tail, shown in Fig. 1 .

We generate initial conditions for our 2D models by axisymmetrizing the matter distribution from the SpEC simulation, keeping only the data with density higher than $10^{8} \mathrm{~g} \mathrm{~cm}^{-3}$, as lower density material is not reliably evolved in the general relativistic merger simulation. The axisymmetric baryon density $\tilde{\rho}$ is obtained by integrating the baryon density $\rho$ over a curve $L(r, z)$ of constant coordinate height $z$ and constant cylindrical radius $r$, and then dividing by the proper length of $L$. For other axisymmetric quantities $\tilde{X}$, we use density-weighted averages, i.e.

$$
\tilde{X}(r, z)=\frac{\oint_{L(r, z)} X \rho W \sqrt{g} d l}{\oint_{L(r, z)} \rho W \sqrt{g} d l}
$$

with $W$ the Lorentz factor, and $g$ the determinant of the spatial 3-metric. The axisymmetrized disk used in this work is constructed from $\left(\tilde{\rho}, \tilde{P}, \tilde{v}_{T}^{x}, \tilde{v}_{T}^{y}, \tilde{v}_{T}^{z}\right)$, where $\mathrm{P}$ is the pressure, and $v_{T}^{i}=u^{i} / u^{t}$ is the 3 -velocity of the fluid with respect to the numerical grid ("transport" velocity). We also constructed an axisymmetrized disk from the average temperature $\tilde{T}$, and found no significant differences in the subsequent evolution of the system. We can assess the effect of this axisymmetrization procedure from Figs. 5, 7 and 9 of Foucart et al. 2015 [28, which show the density, velocity, temperature and electron fraction of the fluid in the equatorial plane of the system $20 \mathrm{~ms}$ after merger. The less symmetric variable is the temperature, which varies by up to a factor of 2 at constant radius in the equatorial plane. Other fluid variables are slightly more axisymmetric.

The dynamical ejecta is reconstructed from the evolution of tracer particles initialized from an earlier snapshot of the evolution, at $t=4 \mathrm{~ms}$ after merger. This is early enough that all of the dynamical ejecta material is still within the computational domain, but late enough that a Newtonian ballistic and adiabatic approximation for the subsequent evolution of the particles is acceptable (see e.g. [6], where smoothed particle hydrodynamics simulations of the tidal ejecta of a BH-NS merger were performed with and without the inclusion of pressure terms, with little differences in the evolution of the ejecta). Using these assumptions we determine the location of the bound and unbound components of the dynamical ejecta at $t=20 \mathrm{~ms}$ after merger, and axisymmetrize that mass distribution. For this component we only consider matter which is located at a radius $r \gtrsim 9 G M_{\mathrm{BH}} c^{-2}$ when the particles are introduced. This includes all of the dynamical ejecta material which leaves the SpEC computational grid during the post-merger evolution. To limit the error introduced by the transition from a general relativistic simulation to Newtonian trajectories, we 
determine the velocity of the tail material by keeping constant the location, direction of motion, and specific energy of the particles. The latter is estimated using the approximation $1+(v / c)^{2} / 2-G M_{\mathrm{BH}} /\left(r c^{2}\right)=-u_{t}$, where $u^{\mu}$ is the 4-velocity of the fluid in the general relativistic simulation.

The disk data is mapped onto a pseudo-Newtonian potential for long-term evolution $(2.2$ by interpolating the fluid density, pressure, electron fraction, and velocity, with the remaining variables obtained with the equation of state selfconsistently. For the dynamical ejecta, the same method is used except that the pressure - which is dynamically unimportant and not extremely reliable given the adiabatic and ballistic evolution of particles - is replaced by the entropy of the matter distribution, from which the temperature is obtained. Regions of very low particle density are removed from the mapping, with the resulting composite mass distribution having nearly constant height-to-radius ratio (Figure 1).

Any regions in the dynamical ejecta that overlap the disk distribution are removed, and an additional cut in radius at $r_{\text {cut }}=260 \mathrm{~km}$ is made to prevent spurious jumps in the density distribution when combining the two components, resulting in a gap of $\sim 50 \mathrm{~km}$ in radius between disk and dynamical ejecta. This gap is filled on a timescale of $\sim 10^{-4}$ s once evolution begins, and its introduction does not have a significant outcome in the evolution. For both components, the interpolation is performed after shifting the radial coordinate by $0.5 r_{g}$ everywhere, which yields a disk mass that agrees well with that computed in the GR simulation. A similar shift was used in [6] to provide a better agreement between GR and Newtonian potentials when mapping data from SpEC simulations of a BH-disk system in the damped harmonic gauge to SPH simulations using a Paczynski-Witta potential.

The baseline configuration obtained in this way has a central BH mass of $8.071 M_{\odot}$ and spin $\chi=0.861$, disk mass $0.06 M_{\odot}$, gravitationally bound dynamical ejecta mass $0.038 M_{\odot}$ (hereafter called fallback) and unbound dynamical ejecta mass $0.075 M_{\odot}$ (hereafter called unbound tail). The degree of gravitational binding is computed relative to the pseudo-Newtonian potential. Passive scalars are assigned to each of these components in order to track their evolution and interaction as a function of time (Figure 1). Additional models are evolved which scale the dynamical ejecta mass or remove it altogether, as described in $\$ 2.5$

\subsection{Time-dependent hydrodynamics}

We solve the time-dependent hydrodynamic equations using FLASH3 [50, 51] in axisymmetric spherical polar coordinates $(r, \theta)$. The public version of the code has been modified to include the physics required to model the long-term evolution of compact object merger remnants [38, 52, 53. Angular momentum transport is treated with an $\alpha$ viscosity prescription [54, and neutrinos are included via a leakage scheme with lightbulb-type self-irradiation, including only charged-current weak interactions. The equation of state is that of Timmes et al. [55, and gravity is modeled with the pseudo-Newtonian potential of Artemova et al. [56].

The computational domain extends from $r_{\text {in }}=24.3 \mathrm{~km}$, midway between the horizon and the ISCO, until $10^{4} r_{\text {in }}$, covering the full range of polar angles. The radial grid is logarithmic with cell size $\Delta r / r \simeq 3.7 \%$, while the polar grid has 56 cells equispaced in $\cos \theta$, achieving $\Delta \theta=\Delta r / r \simeq 2^{\circ}$ on the equator. We employ standard outflow boundary conditions in radius, and reflecting in polar angle at the axis.

During the first $1 / 10$ of an orbit $\left(\sim 2 \times 10^{-4} \mathrm{~s}\right)$, the system is evolved without 

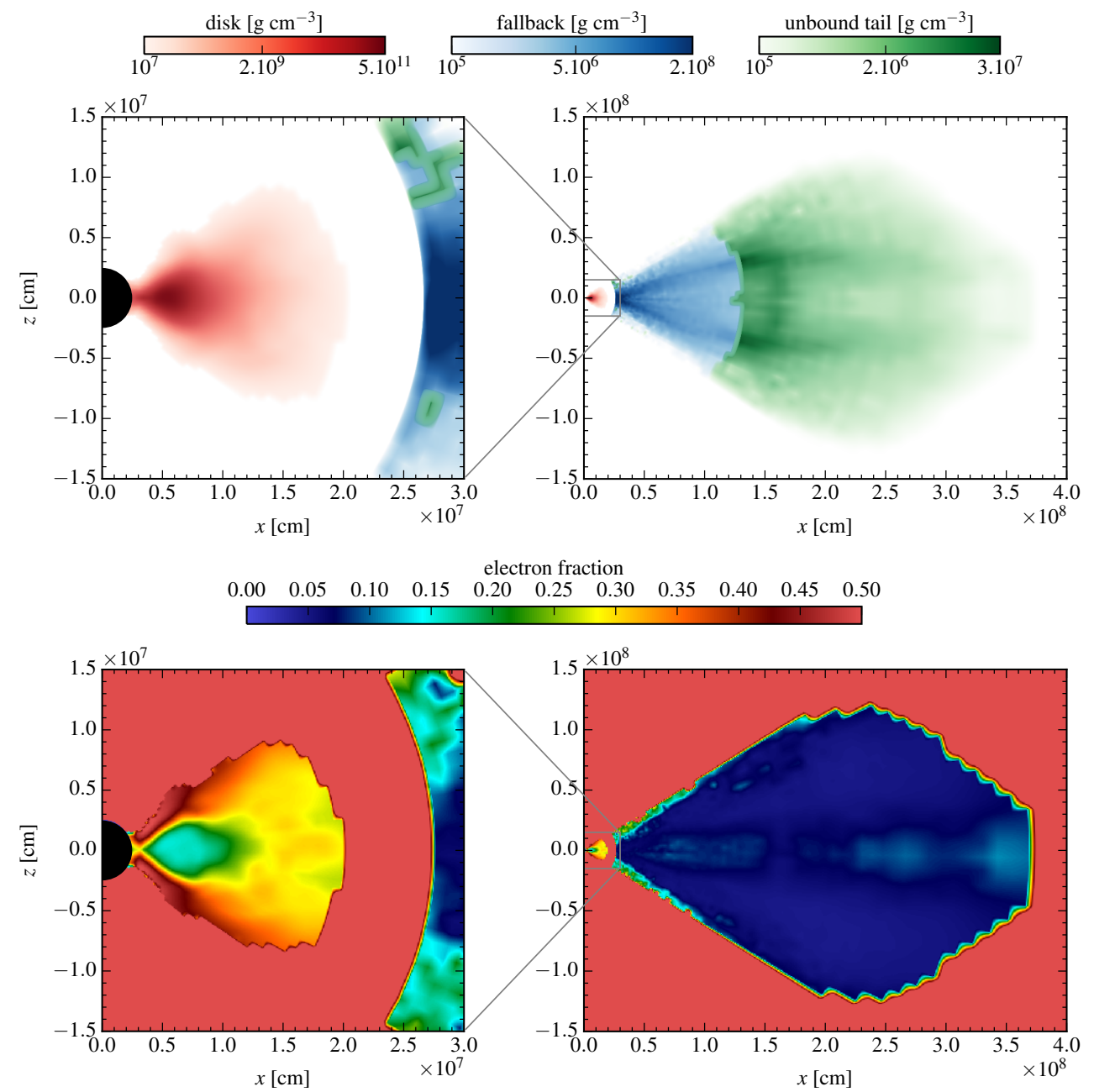

Figure 1: Top: Partial densities ( $=\rho X_{i}$, with $X_{i}$ the mass fraction) of disk, bound dynamical ejecta ("fallback"), and unbound dynamical ejecta ("unbound tail") components at $t=0$ for the baseline model F0. Note that the color scale is not the same for all components; here it is chosen to maximize contrast. Bottom: Electron fraction at $t=0$ in model F0. The dynamical ejecta density is reconstructed from tracer particles and remapped onto the spherical grid. Rough edges are smoothed out by evolving the entire system for $\sim 10^{-4} \mathrm{~s}$ without source terms. The gap between disk and dynamical ejecta - introduced to eliminate overlap between the disk distribution and dynamical ejecta distribution - is also filled within that timescale. 
source terms to allow sharp edges to smooth out and to avoid numerical errors. Subsequently, all source terms are included in the evolution. The ambient density is a power-law in radius, $\rho_{\mathrm{amb}} \propto r^{-2}$, normalized so that it is nearly 10 orders of magnitude lower than the torus density peak $\left(\sim 60 \mathrm{~g} \mathrm{~cm}^{-3}\right)$ at the same location. The density floor is set to be $90 \%$ of the initial ambient density. As time elapses, the floor is gradually decreased interior to $200 \mathrm{~km}$ until it becomes constant and equal to $10 \mathrm{~g} \mathrm{~cm}^{-3}$. Source terms are suppressed when the density is less than 10 times higher than the floor.

In order to generate homologously expanding ejecta for radiative transfer calculations, the material flowing out is sampled at $r_{\text {map }}=10^{9} \mathrm{~cm}$ throughout the simulation. Following the approach of [40] and [41, the sampled material is injected into a new computational domain with an inner radius at $r=r_{\text {map }}$ and outer radius $r=10^{5} r_{\text {map }}$. In these new hydrodynamic simulations, material is evolved without energy source terms except for radioactive heating from $r$-process nucleosynthesis [24, and with a much lower ambient density, $10^{-10} \mathrm{~g} \mathrm{~cm}^{-3}$. By the time the forward shock reaches $r=3 \times 10^{12} \mathrm{~cm}$, the velocity profile is proportional to radius and the kinetic energy is dominant in most of the material.

\subsection{Nucleosynthesis}

For each simulation, $10^{4}$ tracer particles are inserted in the domain, with random positions that follow the mass distribution (i.e., all particles represent the same amount of mass). Thermodynamic trajectories as a function of time are then generated from each particle by interpolating the corresponding variables from the grid at each time. In addition to density, temperature, and composition, we sample neutrino and viscous source terms.

Nucleosynthesis calculations on each trajectory are carried out with the nuclear reaction network code SkyNet [57. The network includes 7843 isotopes up to ${ }^{337} \mathrm{Cn}$. Forward strong rates are taken from the JINA REACLIB database [58], with inverse rates computed assuming detailed balance. Spontaneous and neutron-induced fission rates are taken from [59, 60], 61], and 62. Most weak rates come from 63], 64, and 65, or otherwise from REACLIB. Nuclear masses and partition functions are taken from REACLIB, which contains experimental data where available or FiniteRange Droplet Macroscopic model (FRDM, e.g. 66]) values otherwise. The network evolves the temperature taking into account source terms due to nuclear reactions as computed by SkyNet, as well as viscous heating and neutrino interactions (charged current emission/absorption on free nucleons) from the thermodynamic trajectories.

Computations for each trajectory start from nuclear statistical equilibrium (NSE). Most particles that initially reside in the disk reach temperatures $T \geq 10^{10} \mathrm{~K}$, and their nucleosynthesis is computed from the last time they reach this temperature. Most dynamical ejecta particles have temperatures lower than $3 \times 10^{9} \mathrm{~K}$ at all times, making computation of NSE infeasible (and a bad approximation). We therefore ignore all particles for which the maximum temperature is less than $5 \times 10^{9} \mathrm{~K}$, and instead set their abundance to the average final yield (at a time $10^{13} \mathrm{~s}$ ) of model M14-7-S8 from [6]. That calculation uses the same GR merger simulation to produce tracer particles only from dynamical ejecta material, and starts the evolution at an earlier time such that particles satisfy $T \geq 5 \times 10^{9} \mathrm{~K}$. For the remaining particles, for which the maximum temperature satisfies $5 \leq\left(T / 10^{9} \mathrm{~K}\right) \leq 10$, nucleosynthesis starts from the time at which the maximum temperature is reached. The network calculations performed 
here extend to a time $1 \times 10^{9} \mathrm{~s}$, which is sufficiently long to obtain relatively stable abundances as a function of mass number $A$ except for the heaviest nuclei $(A>210)$ undergoing alpha decay and fission. Since the disk contribution above $A=130$ is subdominant, we consider this to be a reasonable approximation (we ignore elemental abundance changes as a function of $Z$ that take place at times longer than $10^{9} \mathrm{~s}$ ).

\subsection{Radiative transfer}

We calculate synthetic light curves and spectra of the models using the Monte Carlo radiative transfer code SEDONA 67]. The calculations use the axisymmetric homologous ejecta profiles from the hydrodynamical calculations described in $\$ 2.2$, with a setup similar to [18] and [40. The broadband opacity of $r$-process elements is approximated statistically based on the atomic data of [16], and using the $Y_{e}$ from the hydrodynamical simulations as a proxy for the composition. This is a fair approximation given the very sensitive dependence of the production of heavy r-process in $Y_{e}$ [40, 57. We use $Y_{e}<0.25$ as a threshold for the production of lanthanides.

The radiative transfer calculations assume local thermodynamic equilibrium for the atomic level populations and ionization state. We use the average time-dependent radioactive heating rate from [12 for all points of the ejecta except those with $Y_{e}>0.4$, which we asssume have zero heating. We assume $100 \%$ thermalization of the radioactive decay products - in reality, non-efficient thermalization will reduce the kilonova luminosity, especially for times after peak 21. We compute wavelengthdependent light curves for 20 polar angles, equally-spaced in their cosine, over the range 0 to $\pi$. We average the results over three wavelength regions: blue optical $(3500-5000 \AA)$, red optical $(5000-7000 \AA)$, and infrared $(1-3 \mu \mathrm{m})$ to construct the specific luminosity in different bands.

\subsection{Models evolved}

Table 1 summarizes the properties of the eight models computed in this study. The first group includes a simulation of the disk (Fdisk) or dynamical ejecta alone (Fdyn), as well as a case of disk alone with $Y_{e}=0.1$ enforced initially (Fdisk-Y0.1) - to test the sensitivity of the wind composition to initial conditions $\left(Y_{e}=0.1\right.$ is a common choice for the initial composition in post-merger simulations starting from idealized quasi-equilibrium tori, e.g. 38] - and a model of disk alone with no energy source terms (Fdisk-ns), to quantify the contribution of initial transients to the mass ejection. In all models, a viscosity parameter $\alpha=0.03$ is used (c.f. 38), and the initial black hole mass and spin are used in the pseudo-Newtonian potential and kept constant in time.

The second group of models evolves the combined dynamical ejecta plus disk. The fiducial case (F0) simulates the system with the physical parameters from the GR merger simulation, whereas the remaining models (Ft0.1, Ft0.3, and Ft3.0) scale the dynamical ejecta mass by factors of $1 / 10,1 / 3$, and 3 . While the range of scaling factors used here is very wide compared to the numerical error in the determination of the mass of the disk, bound tail, and unbound tail, they are fairly representative of potential uncertainties in the relative mass of these components when varying the parameters of the binary (i.e., for black hole masses in the range $M_{\mathrm{BH}} \sim[5-10] M_{\odot}$ ). In particular, models which reduce the ratio of the mass of the dynamical ejecta to the mass of the disk are more representative of low-mass BH-NS mergers, for which 
Table 1: Models evolved and summary of results. Columns from left to right show model name, initial masses of disk, fallback, and unbound tail, fraction of the disk and fallback ejected in the wind, total ejected fraction $f_{\mathrm{w} \text {,tot }}$ (eq. 4), mass-weighted radial velocity and electron fraction in the wind (including disk and fallback), mass ejected with $Y_{e}>0.25$, and accreted fractions of disk and fallback.

\begin{tabular}{|c|c|c|c|c|c|c|c|c|c|c|c|}
\hline Model & $M_{\mathrm{d}}$ & $\begin{array}{c}M_{\mathrm{f}} \\
\left(10^{-2} M_{\odot}\right.\end{array}$ & $M_{\mathrm{ut}}$ & $\begin{array}{c}f_{\mathrm{w}, \mathrm{d}} \\
(\%)\end{array}$ & $\begin{array}{l}f_{\mathrm{w}, \mathrm{f}} \\
(\%)\end{array}$ & $\begin{array}{l}f_{\mathrm{w}, \mathrm{tot}} \\
(\%)\end{array}$ & $\begin{array}{l}\bar{v}_{r, \mathrm{w}} / c \\
\left(10^{-2}\right)\end{array}$ & $\bar{Y}_{e, \mathrm{w}}$ & $\begin{array}{c}M_{\mathrm{w}, 0.25} \\
\left(10^{-3} M_{\odot}\right)\end{array}$ & $\begin{array}{c}f_{\text {acc, }, \mathrm{d}} \\
(\%)\end{array}$ & $\begin{array}{c}f_{\text {acc, } \mathrm{f}} \\
(\%)\end{array}$ \\
\hline Fdisk & 6 & 0 & 0 & 8 & $\ldots$ & 8 & 3.9 & 0.35 & 5 & 92 & $\ldots$ \\
\hline Fdisk-Y0.1 & & & & 8 & $\ldots$ & 8 & 3.9 & 0.31 & 4 & 92 & $\ldots$ \\
\hline Fdisk-ns $^{a}$ & & & & 0.5 & $\ldots$ & 0.2 & 18 & 0.22 & 0.07 & 8 & $\ldots$ \\
\hline Fdyn & 0 & 3.8 & 7.5 & $\ldots$ & 12 & 12 & 5.0 & 0.07 & 0 & $\ldots$ & 88 \\
\hline F0 & 6 & 3.8 & 7.5 & 5 & 32 & 15 & 4.4 & 0.24 & 9 & 95 & 68 \\
\hline Ft0.1 & & 0.38 & 0.75 & 8 & 55 & 11 & 3.5 & 0.30 & 6 & 93 & 42 \\
\hline Ft0.3 & & 1.3 & 2.5 & 7 & 48 & 14 & 3.9 & 0.26 & 8 & 94 & 52 \\
\hline Ft3.0 & & 11 & 23 & 5 & 20 & 15 & 4.6 & 0.20 & 8 & 96 & 81 \\
\hline
\end{tabular}

${ }^{a}$ Model Fdisk-ns is evolved without momentum or energy source terms.

the disk mass often is an order of magnitude larger than the mass of the dynamical ejecta (see e.g. Fig.11 of [68]). 团 The scaling factor could be even smaller for lower mass BH-NS mergers $\left(M_{\mathrm{BH}}<5 M_{\odot}\right)$ or NS-NS mergers.

The evolution of the accretion disk and dynamical ejecta including all source terms is carried out for 3000 orbits at the initial density peak, or about $\sim 10$ seconds. This timescale corresponds to several viscous times, and is determined by demanding saturation in the mass ejection as measured at the radius at which the disk outflow is sampled $\left(r_{\text {map }}=10^{9} \mathrm{~cm}\right)$. The second simulation step that uses the sampled outflow and evolves it into homomology is run until about $100-200 \mathrm{~s}$. This time is chosen so that most of the mass distribution achieves homology while satisfying the requirement that the swept up mass (in ambient medium) remains lower than $1 \%$ of the disk ejecta mass.

\section{Dynamics of disk wind and dynamical ejecta}

\subsection{Overview of baseline model}

The initial condition for the baseline model F0 is shown in Figure 1. Passive scalars track the evolution of the disk material as well as the gravitationally bound and unbound parts of the dynamical ejecta (fallback and unbound tail). For clarity, we start by discussing the behavior of models that include only the disk or the dynamical ejecta, and then proceed to describe their combined evolution.

At $t=0$, the electron fraction in the disk and dynamical ejecta is very different. With the exception of very low density regions near its inner edge, most of the dynamical ejecta has $Y_{e} \simeq 0.05$. In contrast, the bulk of the disk has $Y_{e} \simeq 0.15-0.2$, increasing away from the disk midplane. The initial electron fraction of the disk is somewhat higher than that employed in long-term disk simulations that start from equilibrium tori as initial conditions (e.g., [38]).

$\ddagger$ We note that this statement only applies to the ratio of the disk and dynamical ejecta masses. In absolute terms, and at constant black hole spin, low mass black holes are not necessarily unfavorable to the production of unbound material. In fact, for low spins, high mass black holes are unable to disrupt the neutron star and do not produce any ejecta at all [69]. 

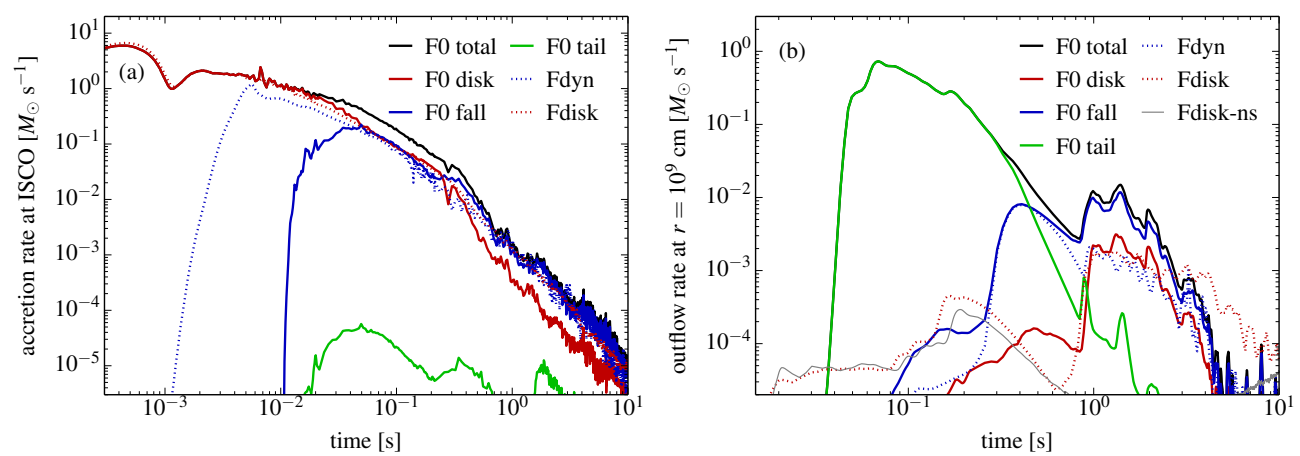

Figure 2: Left: Mass accretion rate at the ISCO for the different components of the baseline model F0 shown as solid lines: disk (red), fallback (blue), and unbound tail (green). For comparison, dotted lines show accretion of disk material for the model without dynamical ejecta (Fdisk, red) and of fallback material for the model without disk (Fdyn, blue). Right: Same as the left panel but now showing total mass ejection at $r=10^{9} \mathrm{~cm}$. The disk-only model without source terms (Fdisk-ns) is shown with a thin grey line.

In the absence of dynamical ejecta (model Fdisk), the disk follows the usual stages of neutrino-cooled disks (e.g., [70]). Two time scales govern the evolution of the system: the orbital time

$$
t_{\text {orb }} \simeq 2\left(\frac{8 M_{\odot}}{M}\right)^{1 / 2}\left(\frac{r}{55 \mathrm{~km}}\right)^{3 / 2} \mathrm{~ms}
$$

where $M$ is the black hole mass, and the viscous time

$$
t_{\mathrm{visc}} \simeq 200\left(\frac{0.03}{\alpha}\right)\left(\frac{0.25}{H / R}\right)^{2}\left(\frac{8 M_{\odot}}{M}\right)^{1 / 2}\left(\frac{r}{55 \mathrm{~km}}\right)^{3 / 2} \mathrm{~ms},
$$

where $H / R$ is the height-to-radius ratio of the disk. Initially, neutrino cooling is important, balancing viscous heating. As the disk evolves and spreads, neutrino emission gradually decreases. At the same time, nuclear recombination adds energy in the outer parts of the disk. By $t=t_{\mathrm{visc}} \simeq 200 \mathrm{~ms}$, the neutrino luminosity has decreased by two orders of magnitude from its initial value, and the disk has reached an advective state, leading to vigorous convection and mass ejection. As shown in Table 1 however, most of the initial disk mass is accreted onto the BH (Figure 22 a), with only $\sim 8 \%$ ejected as a wind. The bulk of mass ejection reaches $10^{9} \mathrm{~cm}$ at times $\gtrsim 1 \mathrm{~s}$ and later (Figure 2b). A small amount of mass due to the non-equilibrium initial condition is ejected on the thermal time $(t \lesssim 30 \mathrm{~ms})$, this outflow is also present in a comparison model evolved without any source terms other than gravity (Fdisk-ns).

In the absence of an accretion disk (model Fdyn), most of the fallback matter moves quickly towards the $\mathrm{BH}$, while the unbound tail moves outward. Both components also expand in polar angle, due to small pressure gradients neglected in the ballistic evolution of the dynamical ejecta material. Initially, the fallback material has nearly constant specific angular momentum, in excess of the value at the innermost stable circular orbit (ISCO) by a factor of $\sim 1.3$. Therefore, the part of this material that falls towards the $\mathrm{BH}$ forms an accretion disk, and the resulting accretion rate at the ISCO evolves in a way similar to the original accretion disk after fallback material has reached the ISCO (Figure 2a). Meanwhile, the unbound tail and part 
of the fallback matter move continuously outward. Over the course of a few orbits, the outward-moving dynamical ejecta establishes a power-law density profile in radius that transitions smoothly between bound and unbound components. A very small amount of unbound tail material is mixed within the fallback at small radius, and moves towards the $\mathrm{BH}$ due to lack of angular momentum. As shown in Table 1, 12\% of the initial fallback mass reaches a radius of $10^{9} \mathrm{~cm}$ (9\% gravitationally unbound), while the rest is accreted. A fraction $99.6 \%$ of the initial unbound tail mass leaves the system.

The early evolution of the combined disk and dynamical ejecta (model F0) is illustrated in Figure 3 . The gap that initially separates the outer edge of the disk and the inner edge of the bound tail is filled on a time $t<t_{\text {orb }}$ by expansion of the disk and inward motion of the bound tail. Fallback matter continues to penetrate the disk on the equatorial plane at later times, with mixing of the two components occurring on a timescale of $\sim 10$ orbits at the disk density peak. For comparison, model Ft0.1 (dynamical ejecta density scaled down by a factor of 10 relative to the baseline model F0) does not experience this violent mixing. Instead, the fallback matter mixes only around the periphery of the disk and does not penetrate regions of higher density. This behavior confirms that the initial gap between disk and dynamical ejecta does not influence the dynamics significantly. Instead, the relevant parameter is the ratio of dynamical ejecta mass to disk mass (which is related to their densities).

A snapshot of the longer-term expansion of the system - compared to the cases in which the disk and dynamical ejecta evolve independently - is shown in Figure 5 a. The bulk of the unbound tail material moves outward identically regardless of whether the disk is present. By $t=t_{\mathrm{visc}} \simeq 200 \mathrm{~ms}$, the fallback material has fully mixed into the original accretion disk, as shown by the similar shape of the density profiles of the two components inside $r \sim 200 \mathrm{~km}$. The original disk material is compressed by the infalling matter, maintaining a sharp density drop at its initial outer edge $(200 \mathrm{~km})$, and a higher density peak relative to the case without dynamical ejecta at the same evolution time. Nevertheless, the very outer edge of the disk has still made its way into the dynamical ejecta, reaching a similar radius $(\sim 2000 \mathrm{~km})$ as model Fdisk, at the same evolutionary time. The disk outflow of model F0 also contains material initially labeled as fallback in higher abundance than disk material outside $r \sim 200 \mathrm{~km}$. These results indicate that (a) with the exception of the outermost regions of the dynamical ejecta, the evolution of which is unaffected by the disk, the fallback and disk components mix efficiently (for model F0), becoming effectively a single disk, and that (b) the wind ejection mechanism is the same as in the case without dynamical ejecta. Changes in the wind properties must therefore be a consequence of the different initial binding energies and masses of the disk and bound dynamical ejecta components.

\subsection{Effect of relative mass of dynamical ejecta and disk}

The overall trends in mass ejection as a function of initial fallback and disk masses are summarized in Table 1 and Figure 6a. Ejection is quantified by the ratio of total ejected mass $\left(M_{w, i}\right)$ at $r=10^{9} \mathrm{~cm}$ to the initial mass $\left(M_{i}\right)$ in disk or fallback components, $f_{w, i}=M_{w, i} / M_{i}$, with $i=\{d, f\}$ for disk or fallback, respectively. We also define a total ejected fraction of disk plus fallback material,

$$
f_{w, t o t}=\frac{f_{w, d} M_{d}+f_{w, f} M_{f}}{M_{d}+M_{f}} .
$$




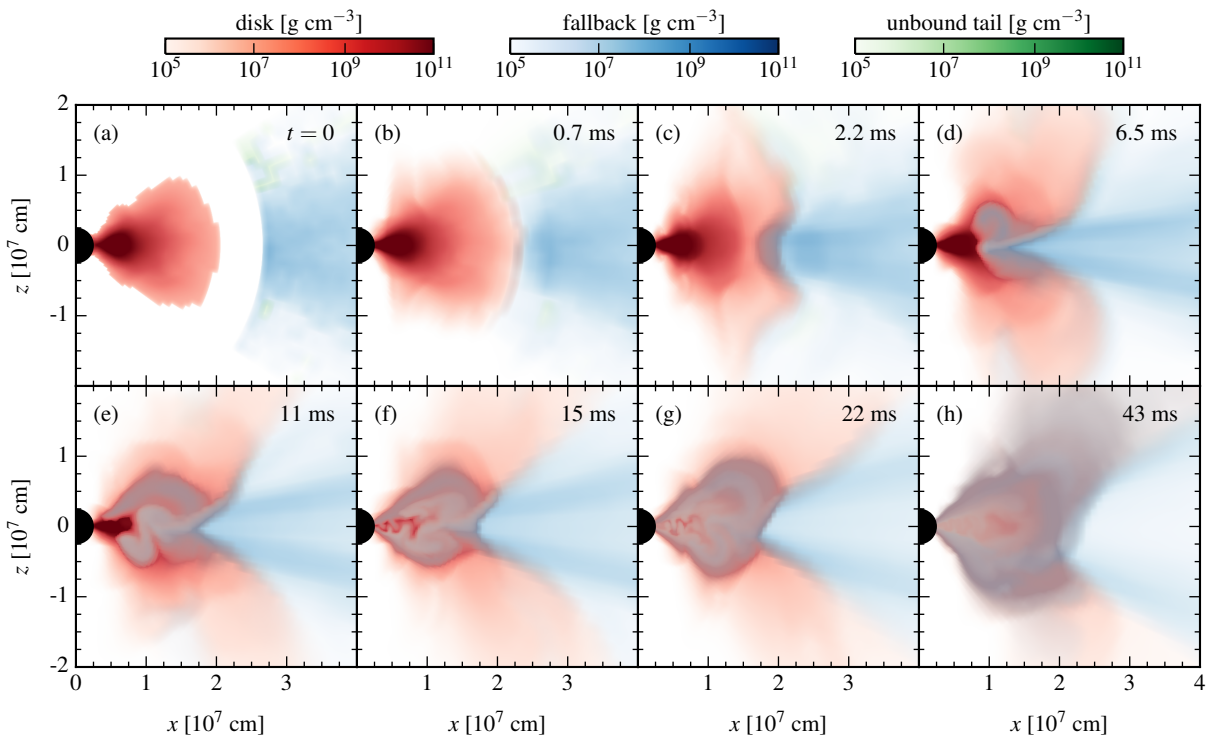

Figure 3: Snapshots of the early evolution of the baseline model F0, showing how the fallback material mixes into the disk. The color maps show partial densities $\rho X_{i}$, with $X_{i}$ the mass fraction of disk, fallback, or unbound tail material, with an opacity that varies linearly from 0 to 1 alongside the color table. Note that the density range for the color scale differs from Figure 1 , here it is the same for all components. The orbital time at the location of the initial disk density peak is $2.2 \mathrm{~ms}$.

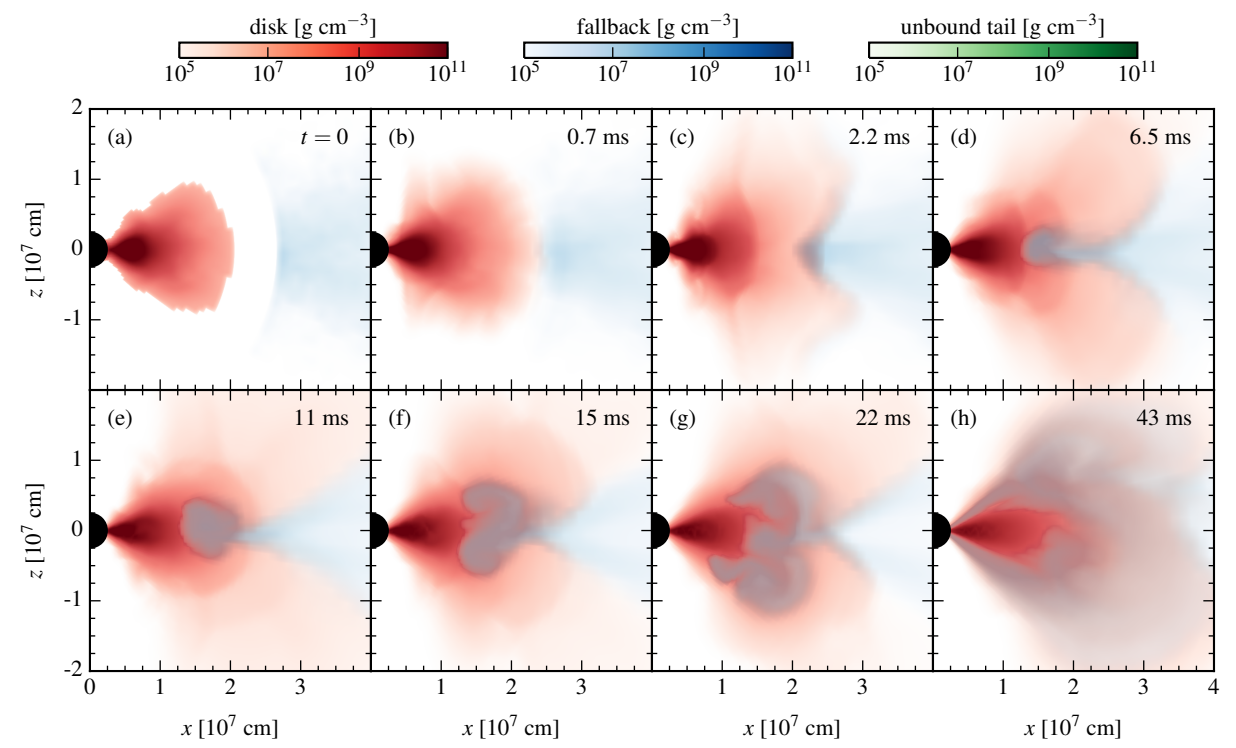

Figure 4: Same as Figure 3, but for model Ft0.1. The fallback matter has a lower density than in model F0, and only mixes in the periphery of the disk. 

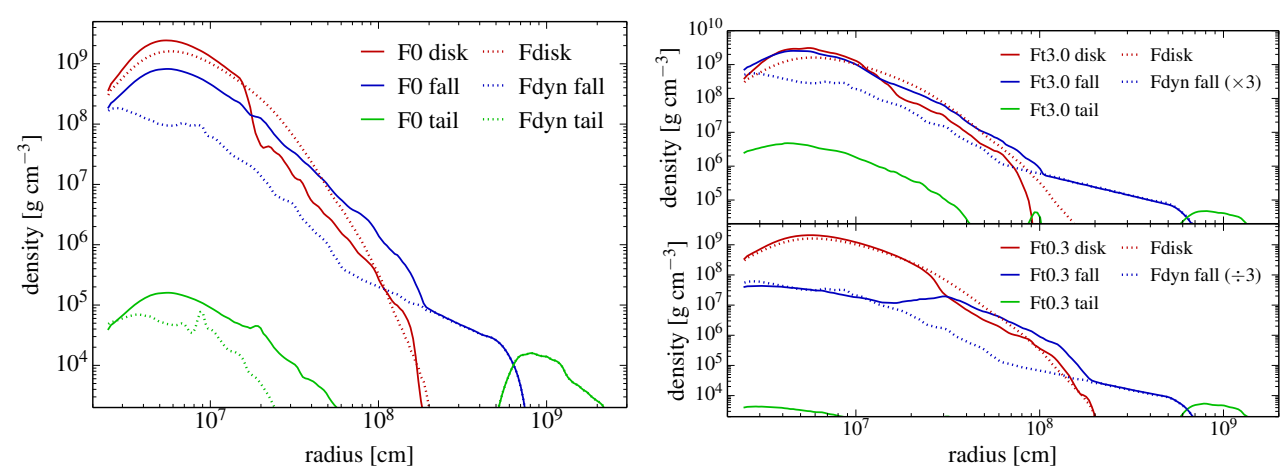

Figure 5: Left: Angle-averaged density profiles for the different components of the diskdynamical ejecta remnant system at $t=216$ ms: disk (red), fallback (blue), and unbound tail (green). For comparison, dotted lines show profiles for the models with disk alone (Fdisk, red) and dynamical ejecta alone (Fdyn, blue and green) at the same time. Right: Same as left panel but for models with scaled dynamical ejecta at $t=216$ ms: Ft3.0 (top) and Ft0.3 (bottom). Models with disk and dynamical ejecta alone (Fdisk and Fdyn, respectively) are shown with dotted lines. The curves from model Fdyn are scaled by the same factor as models Ft0.3 and Ft3.0 (as labeled).

Note that in computing the ejected mass, we include all material that reaches $r=10^{9} \mathrm{~cm}$, irrespective of whether it is gravitationally unbound or not. Equation 4 is equivalent to adding up the mass of disk and fallback material ejected and dividing the result by the sum of initial disk and fallback masses.

The fractions of ejected disk and fallback material both decrease for increasing $M_{f} / M_{d}$, while the total fraction increases to a peak of $f_{w, t o t} \sim 15 \%$ around $M_{f} \sim M_{d}$. This behavior arises from the large fraction of fallback material that is turned around whenever a disk is present $(>20 \%)$, and the relatively weak dependence of the ejected disk fraction on dynamical ejecta mass.

To clarify the dynamics underlying these trends, we show in Figure 5b angleaveraged profiles of disk and fallback densities for models with small and large $M_{f} / M_{d}$ ratios (Ft0.3 and Ft3.0, respectively). Also shown for comparison in each panel is the fallback profile in the model with no disk (Fdyn), scaled to the appropriate dynamical ejecta normalization, and the disk profile in the model with no tail (Fdisk). When the fallback mass is small, bound tail material causes only minor modifications to the radial disk profile, with most of the change occurring in the outer layers. In contrast, for a large initial $M_{f} / M_{d}$ (model Ft3.0), fallback material mixes very effectively into the disk, compressing it significantly relative to the case without dynamical ejecta.

The presence of the disk stops fallback from immediately reaching the $\mathrm{BH}$ and delays the onset of accretion of this material (as in e.g. Figure 2 $\mathrm{b}$ ). This partially accounts for the fact that the ratio of ejected to accreted matter in fallback material increases as $M_{f} / M_{d}$ decreases. Additionally, the (negative) net specific energy of the fluid is initially an order of magnitude larger at the disk density peak than within the bound dynamical ejecta material. The fallback component is thus less gravitationally bound than the disk component, and is relatively easier to eject. 

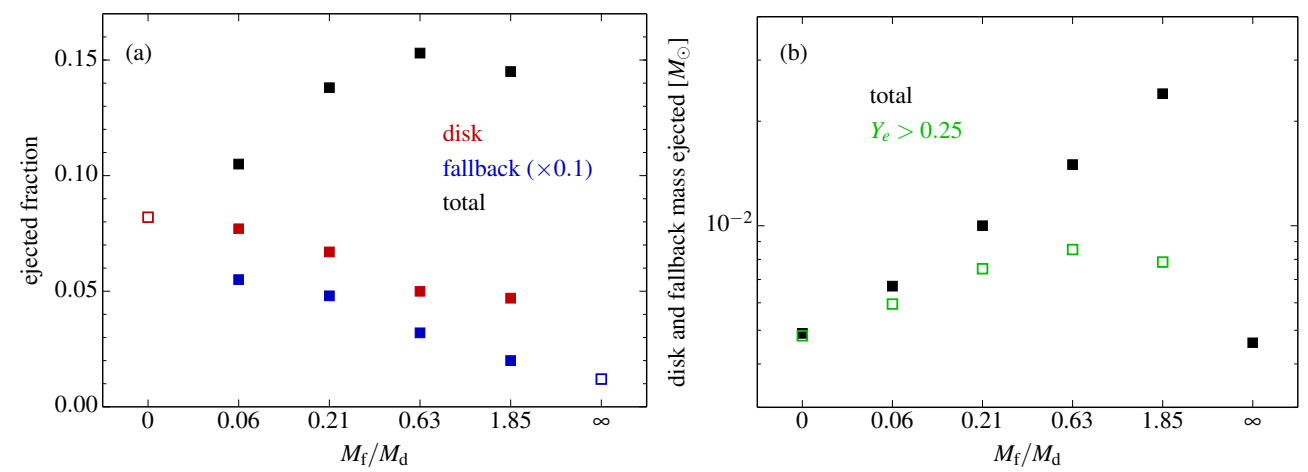

Figure 6: Left: Fraction of the initial mass in disk (red), fallback (blue), and total disk plus fallback material (black, eq. 4) that is ejected as a wind at $r=10^{9} \mathrm{~cm}$, as a function of the ratio of initial fallback to disk masses, for constant initial disk mass (c.f. Table 1). Note that the fraction of fallback matter ejected is scaled down by a factor of 10 to fit in the plot. Right: Total mass (black) and mass with $Y_{e}>0.25$ (green) ejected in the disk wind as a function of the ratio of initial fallback to disk masses. The mass with $Y_{e}>0.25$ is a proxy for the amount of Lanthanide- and Actinide-free mass. See Table 1 for numerical values.

\subsection{Ejecta composition and kinematics}

Table 1 shows the mass-flux-weighted radial velocity and electron fraction of the disk and fallback material ejected at $r=10^{9} \mathrm{~cm}$ for all models evolved. For increasing $M_{f} / M_{d}$, the outflow is faster and more neutron rich. This trend derives from the fact that the fallback is initially very neutron rich and less gravitationally bound (per unit mass) than the disk material. When both components mix, a larger mass-weighted outflow velocity is obtained for fixed energy input per unit mass. Also, material near the transition between bound and unbound dynamical ejecta moves outward and contributes to increase the total outflow momentum.

For a more detailed breakdown of the outflow properties, Figure 7 shows mass histograms of electron fraction and radial velocity for the baseline model F0, and the models without dynamical ejecta (Fdisk) and without disk (Fdyn), for comparison. The bulk of the unbound tail satisfies $Y_{e} \lesssim 0.1$ and is faster than the rest of the outflow, thus evolving independently. In the absence of a disk, the fallback component has a similar electron fraction distribution as the unbound tail, and its velocity distribution is a continuation of the unbound tail distribution to lower velocities. When the disk is present, the fallback material acquires a broad $Y_{e}$ distribution that stretches from the initial unbound tail region to the disk $Y_{e}$ distribution. Material that mixes deep into the disk is subject to weak interactions, acquiring a similar composition as disk material, while matter that remains outermost in radius preserves the original dynamical ejecta composition, with a smooth transition arising from the gradual decrease in strength of weak interactions with increasing radius (temperature). The disk $Y_{e}$ distribution acquires a low- $Y_{e}$ tail stretching to $Y_{e} \sim 0.06$, and the high- $Y_{e}$ cutoff is decreased from 0.45 to 0.4 . The velocity distribution of the fallback material in the baseline model F0 is essentially a scaled-up version of the distribution of the disk component in the same model, again pointing to mixing. The high velocity end is dominated by turned-around fallback matter, which replaces matter at the outer edge of the disk as the fastest and earliest ejecta in the polar direction (relative to model 

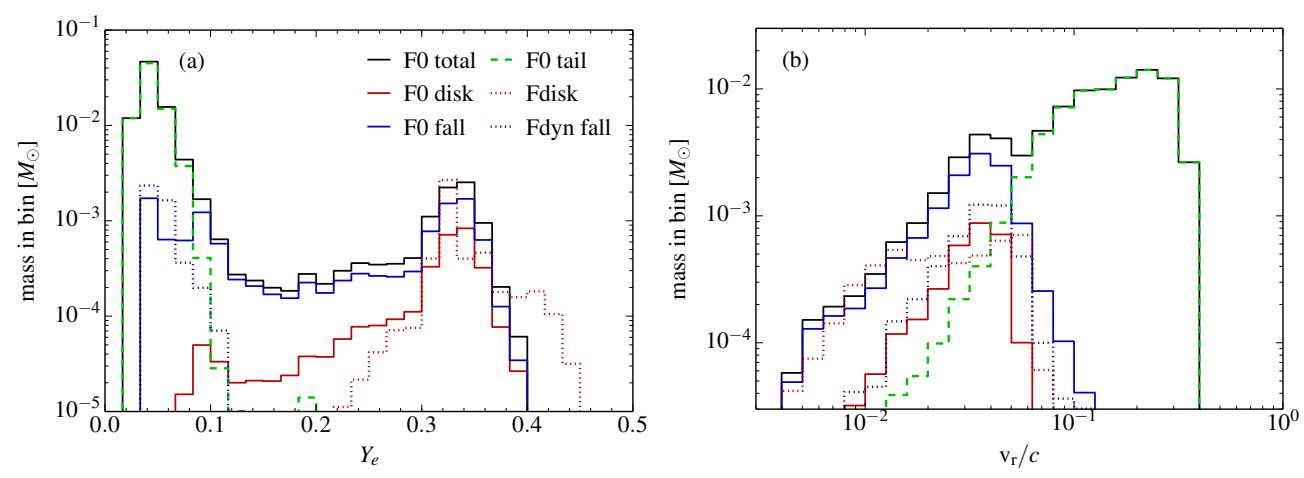

Figure 7: Mass histograms of material ejected at $r=10^{9} \mathrm{~cm}$ as a function of electron fraction (left) and radial velocity (right). Solid lines and dashed lines correspond to the baseline model F0, while dotted lines show the disk-only case (Fdisk, red) and dynamical ejecta-only model (Fdyn, blue), for comparison.

Fdisk). The disk component of model F0 loses the low-end of its velocity distribution relative to model Fdisk. The low-velocity end is now dominated by outward-moving fallback matter far from the bound-unbound transition.

Figure 6b shows ejected mass with $Y_{e}>0.25$, which is indicative of material that cannot form Lanthanides and Actinides and therefore has an optical opacity similar to iron-group elements, thus contributing to an optical kilonova signal (e.g. [40). Even though the outflow becomes on average less neutron rich with increasing $M_{f} / M_{d}$, there is more Lanthanide-free mass ejected until $M_{f} \sim M_{d}$, due to the increasing total mass of the outflows.

Finally, we explore the effect of the initial conditions on the composition of the disk outflow. Figure 8 compares the $Y_{e}$ distribution of two models that evolve the disk alone: one using the electron fraction from the merger simulation (Fdisk), and another one in which $Y_{e}=0.1$ is initially imposed throughout the disk (Fdisk-Y0.1). The latter prescription is adopted in many long-term simulations of disk outflows that begin with equilibrium initial conditions (e.g., [38, 5, 71]). Figure 8 shows that the bulk of the outflow has similar average electron fraction properties in both cases, with mass-flux weighted averages of 0.35 and 0.31 for Fdisk and Fdisk-Y0.1, respectively. Aside from the slightly lower mean value for model Fdisk-Y0.1, its distribution has an extended low- $Y_{e}$ tail and a sharper high- $Y_{e}$ cutoff relative to model Fdisk.

The insensitivity to initial value of $Y_{e}$ can be contrasted with the results of simulations starting from less compact accretion disks, as is obtained in NS-NS mergers. For example, 38 simulated disks with an initial density peak at $r_{\text {peak }} \sim$ $(10-15) G M_{\mathrm{BH}} / c^{2}$, as opposed to $r_{\text {peak }} \sim 5 G M_{\mathrm{BH}} / c^{2}$ here, with the same code and physical model, finding an average electron fraction $Y_{e} \sim 0.15-0.2$. This is significantly different than the results obtained here. The compactness of the disk is determined primarily by the mass of the black hole. General relativistic simulations yield density peaks of the post-merger disk at $r_{\text {peak }} \sim 50 \mathrm{~km}$ (once a mostly axisymmetric disk forms at times $10-20 \mathrm{~ms}$ after merger), without much dependence on the mass of the black hole [72, 73]. Accordingly, more massive black holes have more compact disks.

We conclude that the bulk of the disk outflow acquires its composition from the 

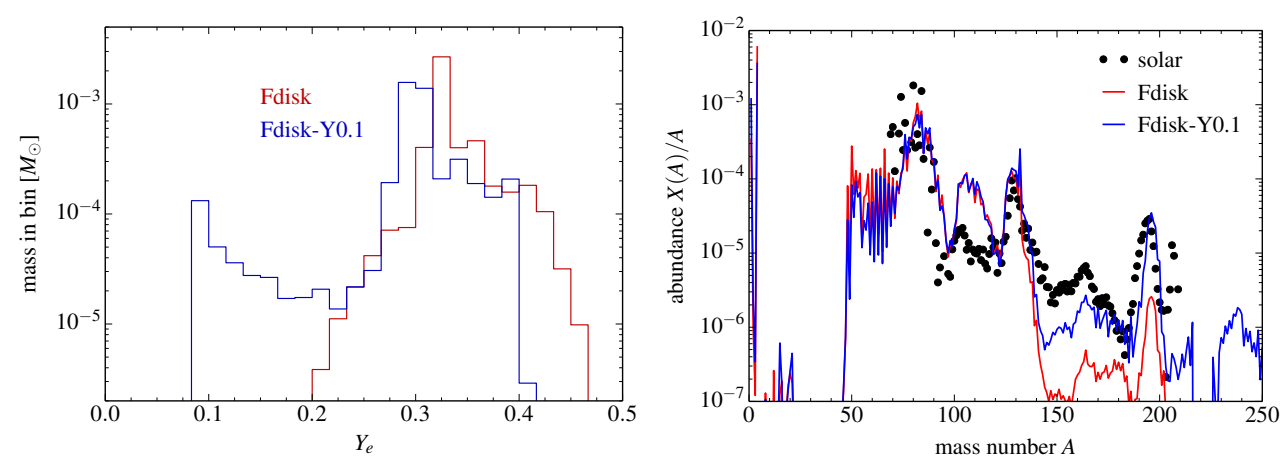

Figure 8: Left: Mass histogram as a function of electron fraction for outflow material measured at $r=10^{9} \mathrm{~cm}$ in disk-only models that use an initial $Y_{e}$ from the merger simulation (Fdisk) or that impose $Y_{e}=0.1$ initially (Fdisk-Y0.1). Right: Nucleosynthesis abundances obtained with thermodynamic trajectories from models Fdisk and Fdisk-Y0.1, normalized so that the mass fractions $X(A)$ integrate to unity. The Solar System abundances from [74] are scaled to model Fdisk at $A=130$.

compactness of the disk, which regulates the temperature and therefore the weak interaction timescale and beta equilibrium $Y_{e}$. Memory of the initial conditions imparts modifications at the $\sim 10 \%$ level in the electron fraction distribution.

\section{Nucleosynthesis}

Figure 9a shows final abundances for model $\mathrm{F} 0$ as a whole and separated by ejecta component. The unbound tail produces heavy $r$-process elements, with a deficit below $A \sim 90$ (c.f. [6]), while the disk outflow generates mostly elements with $A<140$ (Fig. 8b). The bound tail material produces more elements with $A>140$ than the disk material: although part of it undergoes reprocessing by weak interactions upon mixing, it is initially more neutron-rich. Given the relative masses of the ejecta components (Table 1), yields are dominated by the unbound tail for $A \gtrsim 90$. The combined abundance distribution under-produces elements with $A \lesssim 120$, with minor changes relative to the pure dynamical ejecta case (c.f. [6]).

The total abundance distributions for the set of models that varies the dynamical ejecta mass are shown in Figure 9 p. The change in relative contributions from disk, bound tail, and unbound tail lead to about an order of magnitude variation in the abundances below $A=130$ when normalizing to the rare-Earth peak. In particular, model Ft0.1 (smallest dynamical ejecta mass) is the only case that reaches relative abundance values comparable to Solar at the first $r$-process peak $(A=80)$, with good overall agreement elsewhere except for some overproduction at $A=100$. On the other hand, model Ft3.0 (highest dynamical ejecta mass) is hardly different than a case without disk (Fdyn) for $A \gtrsim 70$.

Regarding abundances for the disk alone, Figure $8 \mathrm{~b}$ compares the results for models Fdisk and Fdisk-Y0.1, the latter having $Y_{e}=0.1$ imposed throughout the disk at $t=0$. Abundances below $A=130$ are very similar in both cases, with a ratio of first to second peak abundances that compares well with the Solar System. This robustness below $A=130$ is consistent with previous nucleosynthesis calculations from the disk outflow alone [5, 7]. The low- $Y_{e}$ component in model Fdisk-Y0.1 (Fig. 8a) 

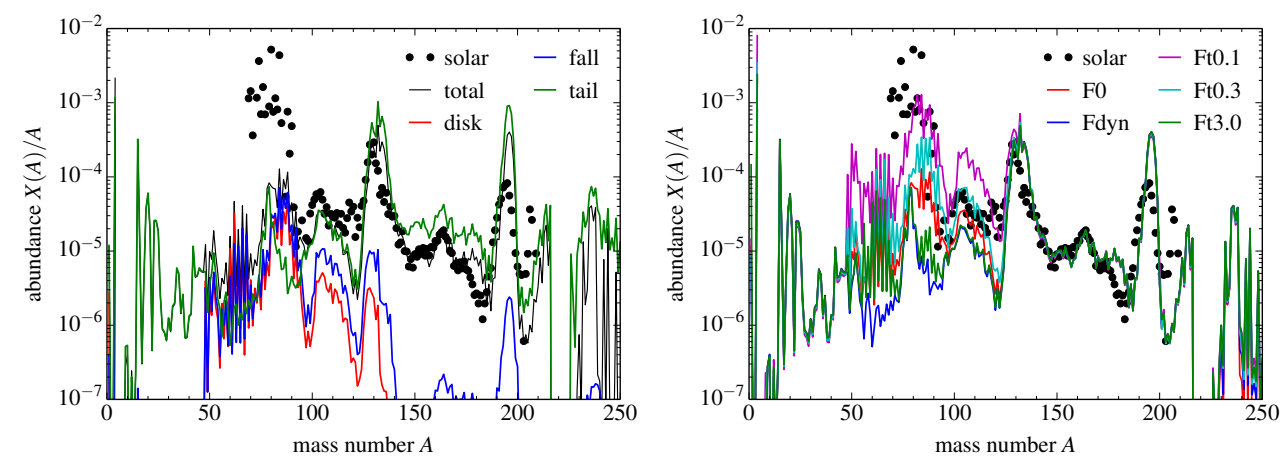

Figure 9: Left: Abundances for model F0 broken down by components and weighted by their relative masses. Right: Combined abundance distributions for models with scaled dynamical ejecta. Model abundance curves and Solar System values are normalized to the rare-Earth peak $(A=164)$ of model F0.

results in a higher production of elements with $A>130$ than model Fdisk, with an excellent ratio of third to second peak abundances and underproduction of rare-Earth elements. Both models display an abundance excess at $A=100$ relative to the Solar System distribution. Also, both cases contain an abundance spike at $A=132$ which is related to the convective character of the disk outflow and an incomplete treatment of nuclear heating in the simulations [7].

\section{Kilonova Signature}

Figure 10 shows the synthetic kilonova light curves for model F0. The bolometric luminosity peaks $\approx 5$ days after the merger. The emission at the earliest times ( $t \lesssim 1$ day) is largely at optical wavelengths, but the spectral energy distribution (SED) reddens quickly thereafter. Over the majority of the light curve duration, the radiation emerges primarily in the near-infrared. The emission in model F0 is generated mainly in the dynamical ejecta, which has a mass an order of magnitude greater than that of the disk wind. The small amount of $Y_{e}>0.25$ (Lanthanidefree) wind ejecta has only a minor impact on the light curves. Figure 10 shows that this high- $Y_{e}$ material is surrounded by lanthanide-rich material in all directions (the high- $Y_{e}$ 'jet' along the polar axis has very low density).

The spectral evolution of the F0 model (shown in Figure 11) helps clarify the broadband light curve behavior. At early times $(t=0.5$ days) the entire ejecta on the grid is heated to temperatures $\gtrsim 5000 \mathrm{~K}$ and - despite the high opacity from lanthanides - the emergent spectra include an optical component. By $t \gtrsim 1$ day, the outermost layers of ejecta have cooled to a photospheric temperature $\approx 2500 \mathrm{~K}$, and the SED has shifted to the near-infrared $(\approx 1 \mu \mathrm{m})$, showing little color evolution thereafter. This evolution of the SEDs is similar to that shown for 1D parameterized models by [16, who also considered how the early optical emission is subject to uncertainties in the lanthanide opacities. In addition, because the photosphere at early times forms in the outermost layers of ejecta, the early optical emission may be sensitive to how well the model resolves the low density surface layers.

The broadband light curves vary by a factor of $\approx 2$ depending on the viewing angle. At most epochs, the kilonova is bolometrically brightest as viewed from 

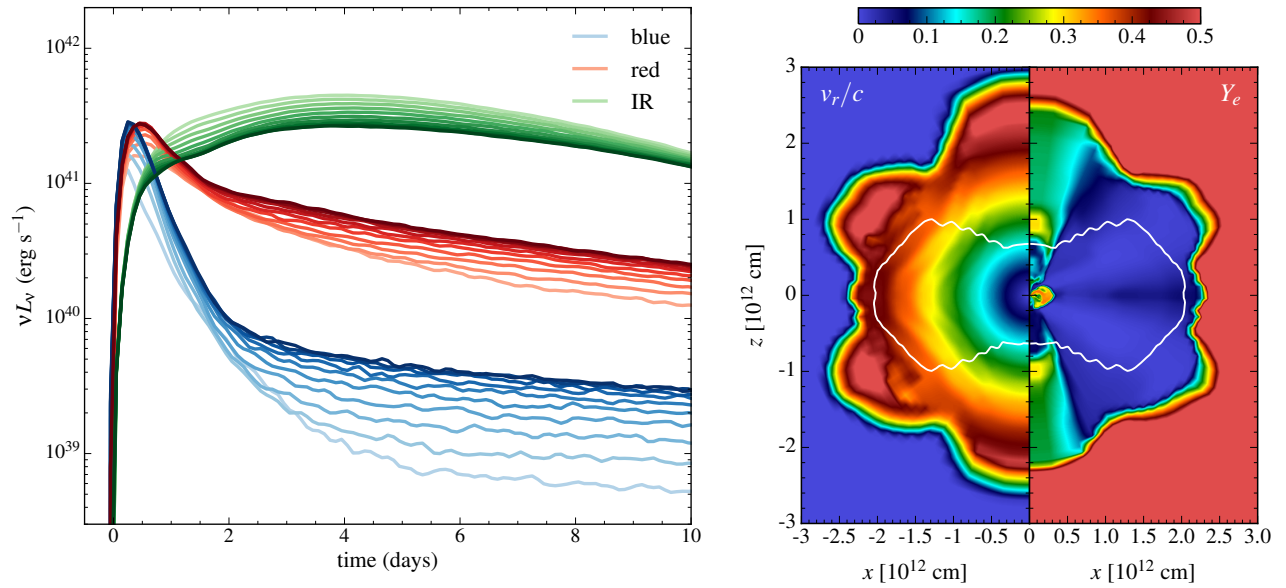

Figure 10: Left: Broadband light curves from model F0 in the wave bands $3500-5000 \AA$ (blue), $5000-7000 \AA$ (red), and $1-3 \mu \mathrm{m}$ (green). For each color, 10 viewing angles equally spaced in their cosine are shown with different shades, spanning the range $\theta=0$ (light, rotation axis) to $\theta=90^{\circ}$ (dark, equatorial plane). Right: Snapshot of the radial velocity (left panel) and electron fraction (right panel) in model F0 at time $t=150 \mathrm{~s}$, when most of the matter distribution has reached homology. This snapshot is used as input for radiative transfer calculations. The white contour corresponds to a density $10^{-6} \mathrm{~g} \mathrm{~cm}^{-3}$.
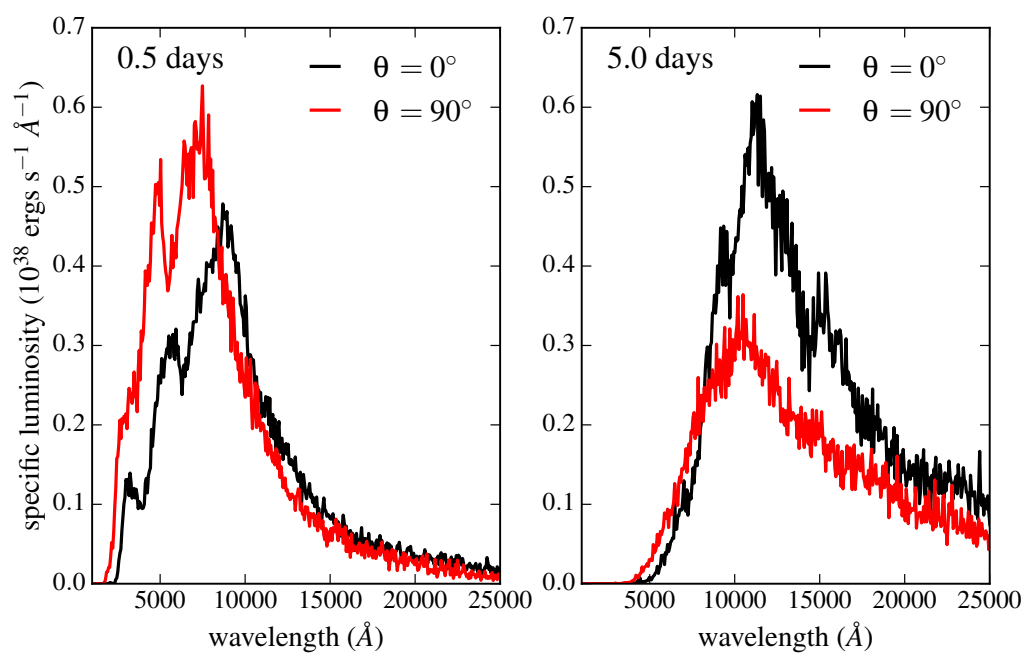

Figure 11: Spectra of model F0 at 0.5 days (left panel) and 5.0 days (right panel) after merger, as observed from both polar $\left(\theta=0^{\circ}\right)$ and equatorial $\left(\theta=90^{\circ}\right)$ viewing angles. The higher equatorial expansion velocity of unbound tail ejecta leads to a greater blueshift of the spectrum for the $\theta=90^{\circ}$ viewing angles. The high frequency variations are Monte Carlo noise. 

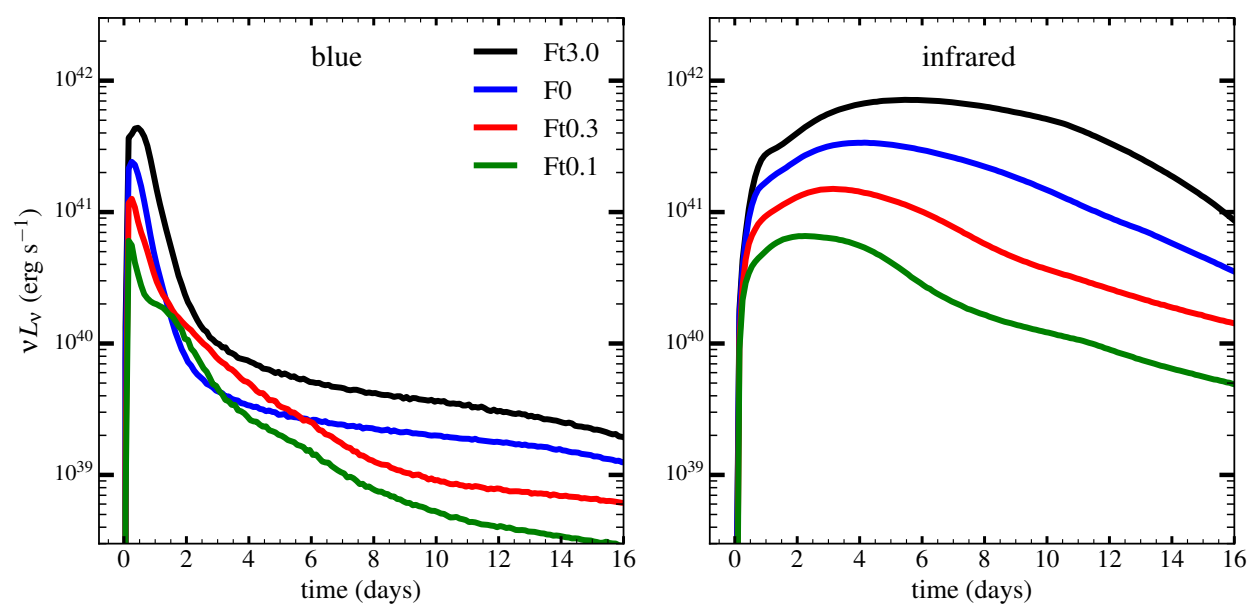

Figure 12: Angle-averaged, broadband light curves from models that scale the dynamical ejecta mass, as labeled. The left panel shows the blue optical band $(3500-5000 \AA)$ and the right panel the infrared band $(1-3 \mu \mathrm{m})$.

directions closer the polar axis $\left(\theta \approx 0^{\circ}\right)$, due to the fact that the projected surface area of the unbound tail is larger by a factor of $\approx 2$ when viewed face-on (Figure 10 ). In the infrared bands, where most of the emission emerges, the light curves follow this geometrical orientation dependence. At optical wavelengths, however, the viewing angle dependence is opposite due to Doppler shift effects. The observed SED is blueshifted by a characteristic outflow velocity of $\approx 0.1 c$, for polar viewing angles and of $\approx 0.3 c$ for equatorial viewing angles (Figure 10 ). Because a greater blueshift moves more of the blue edge of SED into the optical bands, the optical light curves are always brightest from equatorial viewing angles (see Figure 11).

Figure 12 shows the blue and infrared light curves of the entire set of models that scale the dynamical ejecta mass, averaged over all viewing angles. Peak luminosities vary from $6 \times 10^{40} \mathrm{erg} \mathrm{s}^{-1}$ for the dimmest blue optical case to $7 \times 10^{41} \mathrm{erg} \mathrm{s}^{-1}$ for the brightest infrared luminosity. The peak time and luminosity of the infrared emission are monotonic functions of the dynamical ejecta mass, consistent with the larger amount of radioactive material and longer diffusion time. The SEDs and orientation effects of these models are similar to those described for model F0.

Kasen et al. 40] studied the light curves of kilonovae from disk winds, and showed the degree of blue optical emission depended on the amount of lanthanide free $\left(Y_{e} \gtrsim\right.$ $0.25)$ material ejected. They further showed that blue wind emission may be obscured from certain viewing angles if a small mass of lanthanide-containing dynamical ejecta overlaid the wind. In the models of this paper, the lanthanide-free disk ejecta is generally obscured from all viewing angles due to the near complete covering factor of low- $Y_{e}$ unbound tail material (Figure 10). The light curves of these models are therefore mostly insensitive to the amount of lanthanide-free ejecta. As discussed above, the optical luminosity arises from the lanthanide-containing dynamical ejecta, which is hot enough at early times to include some optical component. 


\section{Summary}

We have investigated the long-term evolution of the remnant system left behind after a BH-NS merger, and its electromagnetic and nucleosynthetic signatures. Using initial conditions from a GR merger simulation with neutrino transport, the remnant components are evolved in axisymmetry, accounting for the dominant nuclear and neutrino source terms. Nucleosynthesis is computed from passive tracer particles injected in the simulation, and optical/infrared kilonova light curves are generated using a Monte Carlo radiative transfer code once the ejecta has reached homologous expansion. We have characterized the dynamics of the system and its observable signatures when the relative initial masses of disk and dynamical ejecta are varied. Our main results are the following:

1. - The presence of the disk prevents fallback material from directly reaching the $\mathrm{BH}$. Instead, fallback can mix efficiently into the disk, increasing the effective disk mass and decreasing the net specific binding energy (Fig. 5). The resulting disk outflow is faster and more neutron-rich as the dynamical ejecta mass increases, at fixed disk mass (Table 1). The unbound dynamical ejecta evolves independently of the other components.

2. - The total amount of mass ejected in the disk wind outflow increases as the dynamical ejecta mass increases - at fixed disk mass - up to the point where the bound dynamical ejecta mass and disk mass are approximately equal at $t=0$ (Fig. 6). The mass in Lanthanide- and Actinide-free composition also increases up to this point.

3. - The composition of the disk outflow is determined primarily by the initial compactness of the disk (mass of the BH divided by the radius of the disk density peak). The compactness sets the strength of the gravitational field, and therefore the disk temperature, the weak interaction timescale, and the beta equilibrium $Y_{e}$ of the disk. Memory of the initial composition contributes at the $\sim 10 \%$ level (Fig. 8).

4. - The nucleosynthesis output in the model mapped from the relativistic merger simulation (F0) is dominated by the unbound tail ( $A \gtrsim 130)$, with a sub-dominant contribution from the disk outflow and turned-around fallback matter at $A \lesssim 130$. Good agreement with a Solar $r$-process distribution is obtained for small dynamical ejecta masses (model Ft0.1), more suitable for small BH masses (Fig. 9). Excess production of elements around $A=100$ and at $A=132$ is obtained.

5. - The kilonova signature produces optical emission for the first day after merger, then evolves to the infrared. The peak luminosities are of order $10^{41} \mathrm{erg} \mathrm{s}^{-1}$, with a variation of a factor $\sim 2$ obtained for different viewing angles (Fig. 10). The optical lightcurves are brightest when the system is viewed edge-on, due to Doppler shift effects. The peak optical and infrared luminosities, as well as the transient duration, are monotonically increasing functions of the ratio of initial dynamical ejecta to disk masses (Fig. 12), or equivalently of the total ejected mass. The detailed properties of the early optical emission are sensitive to the outer edges of the disk and dynamical ejecta, and thus will depend on how well resolved are low-density regions in simulations. Nevertheless we consider the general properties of our results are robust.

Our results on the long-term ejecta dynamics of the model with physical 
parameter (F0) differ from our previous study, which employed initial conditions from a Newtonian merger simulation (Fernández et al. 2015; Ref. [41). Results from that work showed only minor mixing between fallback and disk material in the outer parts of the disk outflow (see e.g. Figure 3 in that paper and the insensitivity of the electron fraction of the disk outflow to the presence or absence of dynamical ejecta in their Table 2). This difference can be attributed to the different relative masses of disk and bound dynamical ejecta, and on the way the initial conditions are obtained here. In our model F0, the ratio of fallback-to-disk masses is $0.038 / 0.060$, whereas in the baseline model C2d of Ref. [41] this ratio is $0.02 / 0.20$. The bound dynamical ejecta reaches a peak density a factor of $\sim 16$ lower in $\mathrm{C} 2 \mathrm{~d}$ than in our model $\mathrm{F} 0$, hence it is not able to penetrate the disk and mix in efficiently (in contrast to our Figure 3). A similar behavior is observed in model Ft0.1, for which the bulk of the disk remains unaffected by fallback material, which only mixes in on its periphery (Figure 4). Still, model Ft0.1 has a mean $Y_{e}$ of the disk outflow (0.30) that is lower by $14 \%$ relative to the model without dynamical ejecta (Fdisk, 0.35 ), whereas the corresponding fractional decrease in the models of Ref. 41] is about half $(0.29$ to 0.27 , respectively). This lower mixing efficiency could result from a number of factors, including a higher concentration of dynamical ejecta in the equatorial plane in the general relativistic simulation, and on the fact that the disk and dynamical ejecta used by Ref. 41 are not computed separately, hence some mixing is already built into it (the mean $Y_{e}$ of the resulting outflow compares favorably to the average between our models Ft0.1 and Ft0.3, which bracket the initial fallback-to-disk mass ratio in model C2d of [41]).

An important observational implication of our results is that a substantial amount of blue optical emission can still be generated by the Lanthanide-rich ejecta at early times when the temperatures are high. The duration of this signal is $\lesssim 1$ day, requiring a shorter cadence for its detection. A similar early optical component was seen in the Lanthanide-rich wind models of 40. The robustness of the predicted optical emission is subject to uncertainties in the incomplete Lanthanide atomic data [16; further radiative transfer calculations and atomic structure models are needed to fully assess the early time kilonova colors. A peak optical luminosity of $10^{41} \mathrm{erg} \mathrm{s}$ corresponds to an absolute magnitude $M_{B} \simeq-14$ (apparent magnitude $m_{B} \simeq 24.2$ at $400 \mathrm{Mpc}$ ).

The general properties of our light curves compare favorably to previous work on kilonova emission from BH-NS mergers. Roberts et al. [12] showed that the asymmetrical distribution of dynamical ejecta from NS-NS mergers leads to viewing angle dependences with the brightness varying by a factor of $\approx 2$. A similar magnitude of viewing angle effects was found by [75]. Kyutoku et al. [76] emphasized the asymmetries intrinsic in the BH-NS merger dynamical ejecta due to the dominance of tidal forces on the ejection. They also estimated a factor of a few variation in the kilonova light curve properties at peak as a function of viewing angle. Tanaka et al. 77. carried out multi-dimensional Monte Carlo radiative transfer, and showed that in addition to variations in the line of sight, the asymmetries in the dynamical ejecta cause the emission to be systematically bluer than in the case of NS-NS mergers. In contrast to their results, however, we find that asymmetries due to the line of sight persist beyond 10 days, particularly in the optical band. Finally, Kawaguchi et al. 78. conducted a parameter space study of kilonova properties from BH-NS mergers by developing a fitting formula for the dynamical ejecta mass and for the kilonova light curves as a function of binary mass ratio, BH spin, and NS size (EOS). Our scaling of dynamical ejecta mass at fixed disk mass is a reasonable approximation to 
the variation with $\mathrm{BH}$ spin, for fixed EOS.

Our results are consistent with our previous work 41 when it comes to the source of late-time accretion onto the compact object. Figure 2 shows that accretion of 'fallback' material follows the same time dependence as accretion of disk material, with the slope set by disk physics rather than the usual $\sim t^{-5 / 3}$ obtained when assuming a flat energy distribution of material in Keplerian orbits [79, 80, 81]. A reliable prediction of this time-dependence requires carrying out disk simulations in magnetohydrodynamics (MHD). For instance, the normalization and time-dependence of the disk accretion that we obtain in our models (e.g., Figure 11 of 41]) would imply insufficient accretion energy as required to power the X-ray emission from GRB 130603B at a time of $\sim 1$ day [82. However, relatively small changes in the time exponent could bring the energy production into agreement. Whether the Xrays themselves contribute to the powering of the kilonova, as proposed by [83, is a separate question that depends on the degree of beaming of the highly super-Eddington emission obtained 14. Pursuit of these questions is beyond the scope of this study.

Improving the reliability of these calculations would require the following: (1) general-relativistic merger simulations that employ a larger computational domain, so that initial conditions for both disk and dynamical ejecta are obtained self-consistently, and which use higher resolution so that low-density regions are more reliably evolved (2) long-term general-relativistic, MHD simulations in 3D to evolve both the disk and dynamical ejecta on a fixed metric and with a more realistic geometry, including the dominant neutrino and nuclear source terms, and (3) more reliable opacities for $r$ process elements, which when combined with coupling to the actual composition of the outflow (instead of just $Y_{e}$ ), would yield spectral predictions alongside broadband light curves. The ejecta and disk properties can be sensitive to parameter dependencies other than just the mass ratio, such as the rotation of the neutron star (e.g., 84]) or effects due to non-circularity of the binary (such as e.g., formation in dynamical captures, 85). The development of open-source radiative transfer codes would also allow end-to-end studies to be carried out by a wider community; to our knowledge, the only open-source tool available to date is SNEC [86. Further improvements in the calculations will reveal whether the trends found here are robust.

\section{Acknowledgments}

We thank Kenta Hotokezaka, Masaomi Tanaka, Kunihito Ioka, and Shinya Wanajo for useful discussions. We also thank the anonymous referee for helpful comments that improved the presentation of this paper. RF acknowledges support from the University of California Office of the President, from NSF grant AST-1206097, and from the Faculty of Science at the University of Alberta. Support for this work was provided by NASA through Einstein Postdoctoral Fellowship grant numbered PF4-150122 (FF) awarded by the Chandra X-ray Center, which is operated by the Smithsonian Astrophysical Observatory for NASA under contract NAS8-03060. DK is supported in part by a Department of Energy Office of Nuclear Physics Early Career Award, and by the Director, Office of Energy Research, Office of High Energy and Nuclear Physics, Divisions of Nuclear Physics, of the U.S. Department of Energy under Contract No. DE-AC02-05CH11231. The software used in this work was in part developed by the DOE NNSA-ASC OASCR Flash Center at the University of Chicago. This research used resources of the National Energy Research Scientific Computing Center (NERSC), which is supported by the Office of Science of the U.S. 
Department of Energy under Contract No. DE-AC02-05CH11231. Computations were performed at Edison (repositories $\mathrm{m} 1186$ and m2058). This research also used the Savio computational cluster resource provided by the Berkeley Research Computing program at the University of California, Berkeley (supported by the UC Berkeley Chancellor, Vice Chancellor of Research, and Office of the CIO). We also thank the hospitality of the Yukawa Institute for Theoretical Physics through the workshop Nuclear Physics, Compact Stars, and Compact Star Mergers 2016, during which part of this work was carried out.

\section{References}

[1] Abbott B P et al. 2016 PRL 116061102 (Preprint 1602.03837)

[2] Abbott B P et al. 2016 PRL 116241103

[3] Abadie J et al. 2010 Classical and Quantum Gravity 27 173001-+ (Preprint 1003.2480)

[4] Bauswein A, Baumgarte T W and Janka H T 2013 PRL 111131101 (Preprint 1307.5191)

[5] Just O, Bauswein A, Pulpillo R A, Goriely S and Janka H T 2015 MNRAS 448 541-567 (Preprint 1406.2687)

[6] Roberts L F, Lippuner J, Duez M D, Faber J A, Foucart F, Lombardi Jr J C, Ning S, Ott C D and Ponce M 2017 MNRAS 464 3907-3919 (Preprint 1601.07942)

[7] Wu M R, Fernández R, Martínez-Pinedo G and Metzger B D 2016 MNRAS $4632323-2334$ (Preprint 1607.05290)

[8] Rosswog S 2015 Int. J. of Mod. Phys. D 24 1530012-52 (Preprint 1501.02081)

[9] Fernández R and Metzger B D 2016 Annu. Rev. Nuc. Part. Sci. 6623 (Preprint 1512.05435)

[10] Li L X and Paczyński B 1998 ApJ 507 L59-L62 (Preprint astro-ph/9807272)

[11] Metzger B D, Martínez-Pinedo G, Darbha S, Quataert E, Arcones A, Kasen D, Thomas R, Nugent P, Panov I V and Zinner N T 2010 MNRAS 406 2650-2662 (Preprint 1001.5029)

[12] Roberts L F, Kasen D, Lee W H and Ramirez-Ruiz E 2011 ApJ 736 L21 (Preprint 1104.5504)

[13] Tanaka M 2016 Advances in Astronomy 2016634197 (Preprint 1605.07235)

[14] Metzger B D 2016 preprint, arXiv:1610.09381 (Preprint 1610.09381)

[15] Metzger B D and Berger E 2012 ApJ 74648 (Preprint 1108.6056)

[16] Kasen D, Badnell N R and Barnes J 2013 ApJ 77425 (Preprint 1303.5788)

[17] Fontes C J, Fryer C L, Hungerford A L, Hakel P, Colgan J, Kilcrease D P and Sherrill M E 2015 High Energy Density Physics 16 53-59

[18] Barnes J and Kasen D 2013 ApJ 77518 (Preprint 1303.5787)

[19] Tanaka M and Hotokezaka K 2013 ApJ 775113 (Preprint 1306.3742)

[20] Hotokezaka K, Wanajo S, Tanaka M, Bamba A, Terada Y and Piran T 2016 MNRAS 459 35-43 (Preprint 1511.05580)

[21] Barnes J, Kasen D, Wu M R and Martínez-Pinedo G 2016 ApJ 829110 (Preprint 1605.07218)

[22] Rosswog S, Feindt U, Korobkin O, Wu M R, Sollerman J, Goobar A and Martinez-Pinedo G 2016 preprint, arXiv:1611.09822 (Preprint 1611.09822)

[23] Hotokezaka K, Kiuchi K, Kyutoku K, Okawa H, Sekiguchi Y i, Shibata M and Taniguchi K 2013 Phys. Rev. D 87024001 (Preprint 1212.0905)

[24] Korobkin O, Rosswog S, Arcones A and Winteler C 2012 MNRAS 426 1940-1949 (Preprint 1206.2379

[25] Bauswein A, Goriely S and Janka H T 2013 ApJ 77378

[26] Wanajo S, Sekiguchi Y, Nishimura N, Kiuchi K, Kyutoku K and Shibata M 2014 ApJ 789 L39 (Preprint 1402.7317)

[27] Sekiguchi Y, Kiuchi K, Kyutoku K and Shibata M 2015 PRD 91064059 (Preprint 1502.06660 )

[28] Foucart F, O'Connor E, Roberts L, Duez M D, Haas R, Kidder L E, Ott C D, Pfeiffer H P, Scheel M A and Szilagyi B 2015 PRD 91124021 (Preprint 1502.04146)

[29] Palenzuela C, Liebling S L, Neilsen D, Lehner L, Caballero O L, O'Connor E and Anderson M 2015 PRD 92044045 (Preprint 1505.01607)

[30] Lehner L, Liebling S L, Palenzuela C, Caballero O L, O'Connor E, Anderson M and Neilsen D 2016 Classical and Quantum Gravity 33184002 (Preprint 1603.00501)

[31] Radice D, Galeazzi F, Lippuner J, Roberts L F, Ott C D and Rezzolla L 2016 MNRAS 460 3255-3271 (Preprint 1601.02426)

[32] Foucart F, O'Connor E, Roberts L, Kidder L E, Pfeiffer H P and Scheel M A 2016 Phys. Rev. D, in press (Preprint 1607.07450) 
[33] Ruffert M, Janka H T, Takahashi K and Schaefer G 1997 A\&A 319 122-153 (Preprint arXiv:astro-ph/9606181)

[34] Dessart L, Ott C D, Burrows A, Rosswog S and Livne E 2009 ApJ 690 1681-1705 (Preprint 0806.4380

[35] Perego A, Rosswog S, Cabezón R M, Korobkin O, Käppeli R, Arcones A and Liebendörfer M 2014 MNRAS 443 3134-3156 (Preprint 1405.6730)

[36] Metzger B D, Piro A L and Quataert E 2009 MNRAS 396 304-314 (Preprint 0810.2535)

[37] Lee W H, Ramirez-Ruiz E and López-Cámara D 2009 ApJ 699 L93-L96 (Preprint 0904.3752)

[38] Fernández R and Metzger B D 2013 MNRAS 435 502-517 (Preprint 1304.6720)

[39] Kiuchi K, Sekiguchi Y, Kyutoku K, Shibata M, Taniguchi K and Wada T 2015 PRD 92064034 (Preprint 1506.06811)

[40] Kasen D, Fernández R and Metzger B D 2015 MNRAS 450 1777-1786 (Preprint 1411.3726)

[41] Fernández R, Quataert E, Schwab J, Kasen D and Rosswog S 2015 MNRAS 449 390-402 (Preprint 1412.5588)

[42] Foucart F, Deaton M B, Duez M D, O'Connor E, Ott C D, Haas R, Kidder L E, Pfeiffer H P, Scheel M A and Szilagyi B 2014 PRD 90024026 (Preprint 1405.1121)

[43] http://www.black-holes.org/SpEC.html

[44] Lindblom L, Scheel M A, Kidder L E, Owen R and Rinne O 2006 Class. and Quantum Grav. 23447 (Preprint gr-qc/0512093)

[45] Duez M D, Foucart F, Kidder L E, Pfeiffer H P, Scheel M A and Teukolsky S A 2008 Phys.Rev.D 78104015 (Preprint 0809.0002)

[46] Foucart F, Deaton M B, Duez M D, Kidder L E, MacDonald I, Ott C D, Pfeiffer H P, Scheel M A, Szilagyi B and Teukolsky S A 2013 Phys.Rev.D 87084006 (Preprint 1212.4810)

[47] Thorne K S 1980 Rev. Mod. Phys. 52 299-339

[48] Shibata M, Kiuchi K, Sekiguchi Y and Suwa Y 2011 Progress of Theoretical Physics 1251255 (Preprint 1104.3937)

[49] Lattimer J M and Swesty F D 1991 Nucl. Phys. A535 331-376

[50] Fryxell B, Olson K, Ricker P, Timmes F X, Zingale M, Lamb D Q, MacNeice P, Rosner R, Truran J W and Tufo H 2000 ApJS $131273-334$

[51] Dubey A, Antypas K, Ganapathy M K, Reid L B, Riley K, Sheeler D, Siegel A and Weide K 2009 J. Par. Comp. $35512-522$

[52] Fernández R and Metzger B D 2013 ApJ $\mathbf{7 6 3} 108$

[53] Metzger B D and Fernández R 2014 MNRAS 441 3444-3453

[54] Shakura N I and Sunyaev R A 1973 A $\& A$ A $24337-355$

[55] Timmes F X and Swesty F D 2000 ApJS 126 501-516

[56] Artemova I V, Bjoernsson G and Novikov I D 1996 ApJ 461565

[57] Lippuner J and Roberts L F 2015 ApJ 81582 (Preprint 1508.03133)

[58] Cyburt R H, Amthor A M, Ferguson R, Meisel Z, Smith K, Warren S, Heger A, Hoffman R D, Rauscher T, Sakharuk A, Schatz H, Thielemann F K and Wiescher M 2010 ApJS 189 240-252

[59] Frankel S and Metropolis N 1947 Physical Review 72 914-925

[60] Panov I V, Korneev I Y, Rauscher T, Martínez-Pinedo G, Kelić-Heil A, Zinner N T and Thielemann F K 2010 A 6 A 513 A61 (Preprint 0911.2181)

[61] Mamdouh A, Pearson J M, Rayet M and Tondeur F 2001 Nuclear Physics A 679 337-358 (Preprint nucl-th/0010093)

[62] Wahl A C 2002 Systematics of Fission-Product Yields, Technical Report LA-13928 (Los Alamos, NM: Los Alamos National Laboratory)

[63] Fuller G M, Fowler W A and Newman M J 1982 ApJS 48 279-319

[64] Oda T, Hino M, Muto K, Takahara M and Sato K 1994 Atomic Data and Nuclear Data Tables $56231-403$

[65] Langanke K and Martínez-Pinedo G 2000 Nuclear Physics A 673 481-508 (Preprint nucl-th/ 0001018)

[66] Möller P, Nix J R, Myers W D and Swiatecki W J 1995 Atomic Data and Nuclear Data Tables 59185 (Preprint nucl-th/9308022)

[67] Kasen D, Thomas R C and Nugent P 2006 ApJ 651 366-380 (Preprint astro-ph/0606111)

[68] Kyutoku K, Ioka K, Okawa H, Shibata M and Taniguchi K 2015 PRD 92044028 (Preprint 1502.05402)

[69] Foucart F 2012 Phys.Rev.D 86124007 (Preprint 1207.6304)

[70] Metzger B D, Piro A L and Quataert E 2008 MNRAS $390781-797$ (Preprint 0805.4415)

[71] Fernández R, Kasen D, Metzger B D and Quataert E 2015 MNRAS 446 750-758

[72] Foucart F, Duez M D, Kidder L E and Teukolsky S A 2011 Phys.Rev.D 83024005 (Preprint 1007.4203) 
[73] Foucart F, Duez M D, Kidder L E, Scheel M A, Szilagyi B and Teukolsky S A 2012 Phys.Rev.D 85044015 (Preprint 1111.1677)

[74] Goriely S 1999 A\& $A \mathbf{3 4 2} 881-891$

[75] Grossman D, Korobkin O, Rosswog S and Piran T 2014 MNRAS 439 757-770 (Preprint 1307.2943)

[76] Kyutoku K, Ioka K and Shibata M 2013 PRD 88041503 (Preprint 1305.6309)

[77] Tanaka M, Hotokezaka K, Kyutoku K, Wanajo S, Kiuchi K, Sekiguchi Y and Shibata M 2014 ApJ $\mathbf{7 8 0} 31$ (Preprint 1310.2774)

[78] Kawaguchi K, Kyutoku K, Shibata M and Tanaka M 2016 ApJ 82552 (Preprint 1601.07711)

[79] Rees M J 1988 Nature 333 523-528

[80] Rosswog S 2007 MNRAS 376 L48-L51 (Preprint arXiv:astro-ph/0611440)

[81] Metzger B D, Arcones A, Quataert E and Martínez-Pinedo G 2010 MNRAS 402 2771-2777 (Preprint 0908.0530)

[82] Fong W, Berger E, Metzger B D, Margutti R, Chornock R, Migliori G, Foley R J, Zauderer B A, Lunnan R, Laskar T, Desch S J, Meech K J, Sonnett S, Dickey C, Hedlund A and Harding P 2014 ApJ $\mathbf{7 8 0} 118$ (Preprint 1309.7479)

[83] Kisaka S, Ioka K and Nakar E 2016 ApJ 818104 (Preprint 1508.05093)

[84] Dietrich T, Bernuzzi S, Ujevic M and Tichy W 2017 PRD 95044045 (Preprint 1611.07367)

[85] Paschalidis V, East W E, Pretorius F and Shapiro S L 2015 PRD 92121502 (Preprint 1510.03432

[86] Morozova V, Piro A L, Renzo M, Ott C D, Clausen D, Couch S M, Ellis J and Roberts L F 2015 ApJ 81463 (Preprint 1505.06746) 